\title{
Educación superior en Brasil y modalidades de colaboración intercultural - espacio estratégico de construcción de la autodeterminación de los pueblos indígenas
}

\section{Educação superior no Brasil e modalidades de colaboração intercultural - espaço estratégico de construção da autodeterminação dos povos indígenas}

Higher education in Brazil and modalities of intercultural collaboration - strategic space for the construction of self-determination of indigenous peoples

Antonio Hilário Aguilera Urquiza ${ }^{1}$

DOI: http://dx.doi.org/10.20435/serie-estudos.v24i50.1157

\begin{abstract}
Resumen: El presente texto tiene por objetivo presentar una reflexión teórica, a partir de la convivencia con estudiantes indígenas en las licenciaturas específicas y cursos regulares, en las universidades del estado de Mato Grosso do Sul, Brasil. Desde hace tiempo, estos pueblos originarios aceden a la educación superior, pero, en los últimos años, esa presencia es cada vez más significativa. Tenemos, así, una expresiva cantidad de indígenas estudiando grado y posgrado, inseridos en un contexto que provoca inúmeras reflexiones, siendo una de ellas el lugar de los llamados conocimientos tradicionales y las prácticas de colaboración intercultural. Esta relación entre los llamados conocimientos científicos (occidentales, eurocéntricos), y los conocimientos tradicionales serán objetos de estudio teniendo como base el concepto de interculturalidad y de las discusiones de los autores conocidos como poscoloniales. Veremos que las estrategias y modalidades de colaboración intercultural, tienen como meta la construcción de la autonomía y autodeterminación de estos pueblos tradicionales. Todo esto, se presenta como una de las salidas a la superación del que llamamos aquí de universidad monocultural.
\end{abstract}

Palabras clave: pueblos indígenas; interculturalidad; conocimientos tradicionales; colonialidade; autodeterminación.

\footnotetext{
${ }^{1}$ Universidade Federal de Mato Grosso do Sul (UFMS), Campo Grande, Mato Grosso do Sul, Brasil.
} 
Resumo: O presente texto tem por objetivo apresentar uma reflexão teórica, a partir da convivência com estudantes indígenas nas licenciaturas específicas e cursos regulares, nas universidades do estado de Mato Grosso do Sul / Brasil. Há tempos, estes povos originários chegam à educação superior, porém, nos últimos anos, essa presença é cada vez mais significativa. Temos, assim, uma expressiva quantidade de indígenas estudando graduação e pós-graduação, inseridos em um contexto que provoca inúmeras reflexões, sendo uma delas o lugar dos chamados conhecimentos tradicionais e as práticas de colaboração intercultural. Esta relação entre os chamados conhecimentos científicos (Ocidentais, eurocêntricos), e os conhecimentos tradicionais serão objetos de estudo, tendo como base o conceito de interculturalidade e as discussões dos autores conhecidos como pós-coloniais. Veremos que as estratégias e modalidades de colaboração intercultural, possuem como meta a construção da autonomia e autodeterminação destes povos tradicionais. Tudo isto, se apresenta como uma das saídas para a superação do chamamos aqui de universidade monocultural.

Palavras-chave: povos indígenas; interculturalidade; conhecimentos tradicionais; colonialidade; autodeterminação.

Abstract: The present text intends to present theoretical reflection, from the coexistence with indigenous students in the specific bachelor's degrees and regular courses, in the universities of the state of Mato Grosso do Sul, Brazil. For some time now, these indigenous peoples have attained higher education, but in recent years, this presence has become increasingly significant. We have, thus, an expressive amount of indigenous people studying undergraduate and post-grade, a context that provokes innumerable reflections, one of them being the place of the so-called traditional knowledge and intercultural collaboration practices. This relationship between the socalled scientific knowledge (Western, Eurocentric), and traditional knowledge will be the result of an analysis based on the concept of interculturality and the discussions of authors known as post-colonial. We will see that the strategies and modalities of intercultural collaboration have the goal of building the autonomy and self-determination of these traditional peoples. All this, is presented as one of the ways out of which we call here a monocultural university.

Keywords: indigenous peoples; interculturality; traditional knowledge; colonialidade; selfdetermination.

\section{INTRODUCCIÓN}

A lo largo del período colonial constatamos intentos y prácticas educativas direccionadas "para" los pueblos indígenas en América Latina. Inicialmente, los jesuitas pretendían la cristianización a través de la catequesis, utilizando - casi siempre - la propia lengua materna de los pueblos autóctonos en el proceso de traducción de oraciones y de partes de la Biblia, para subsidiar ese objetivo de evangelización y "civilización de los salvajes".

Con base en concepciones eurocéntricas, esas prácticas coloniales de educación y escolarización de los pueblos indígenas desconsideraban casi com- 
Educación superior en Brasil y modalidades de colaboración intercultural - espacio estratégico de construcción de la autodeterminación de los pueblos indígenas

pletamente sus especificidades autóctonas, imponiendo, a su manera, nuevas formas de visión del mundo, organización del tiempo, relación con la naturaleza y el trabajo. Aun después del período histórico de la colonización, esas prácticas de "educación para el indio", como afirma Meliá (1979), siguen en marcha en las aldeas, ora a través de los misionarios, ora a través del propio Estado; en el caso de Brasil, por medio del Servicio de Protección al Indio (SPI) (a partir de 1910) y posteriormente por la Fundación Nacional del Indio (FUNAI).

Pese a las "marcas profundas de desvalorización de las lenguas nativas y de desmantelamiento de organizaciones y prácticas sociales producidas por los muchos años de colonización y colonialidad², la escuela también pasó a hacer parte de la vida de eses pueblos" (BERGAMASCHI; DOEBBER; BRITO, 2018, p. 38), inicialmente como "punta de lanza", para la dominación y apropiación de sus territorios tradicionales y de su universo simbólico y, más recientemente, esa misma escuela pasa a ser resignificada por los pueblos indígenas, como herramienta de lucha política y de autodeterminación, como veremos a continuación.

En la actualidad, cuando se habla en educación escolar indígena, normalmente tenemos por un lado: currículo indígena, profesor indígena, lengua y conocimientos indígenas y, por otro: educación y asignaturas escolares, sistemas de enseñanza, contenidos legitimados en "plan de estudios", etc. Dos lógicas de producción de conocimiento, de lectura de la realidad, que presupone el encuentro de identidades y diferencias que buscan dialogar bajo el paradigma de la interculturalidad y construir un cotidiano escolar para los pueblos indígenas con un nuevo sentido y un nuevo significado. En esa posibilidad de encuentro entre culturas, se produce un nuevo espacio con nuevas complejidades (AGUILERA URQUIZA, 2010). La problemática que se propone para reflexión es: ¿̇en qué medida ese modelo de educación eurocéntrico podrá se abrir para los pueblos indígenas que ingresan a las universidades? O todavía: ¿cómo superar el modelo de universidad monocultural y hacer con que surjan prácticas de interculturalidad?

\footnotetext{
${ }^{2}$ Colonización tiene que ver con el período colonial por el cual pasaron todos los países americanos. Diferente de esta concepción, la colonialidad se refiere al que se quedó en el imaginario y en las prácticas sociales y políticas. Se trata de "um padrão de poder que emergiu como resultado do colonialismo moderno, mas em vez de estar limitado a uma relação formal de poder entre dois povos ou nações, se relaciona à forma como o trabalho, o conhecimento, a autoridade e as relações intersubjetivas se articulam entre si através do mercado capitalista mundial e da ideia de raça" (TORRES, 2007, p. 131 apud CALDERONI, 2016).
} 
Este texto se propone a ofrecer un breve panorama de la relación de los pueblos indígenas con la educación superior, tomando por bases las experiencias en los últimos años, en el estado de Mato Grosso del Sul/Brasil, y la reflexión acerca de la posibilidad de pensar un currículo intercultural, o sea, que pueda incluir los llamados etnoconocimientos y las modalidades de "colaboración intercultural" (MATO, 2017). No tenemos como foco la discusión de las políticas de acción afirmativa de acceso a la educación superior ${ }^{3}$, pero, sobre todo, la permanencia y la posibilidad de la universidad incluir otros conocimientos.

\section{EDUCACIÓN SUPERIOR, PUEBLOS INDÍGENAS Y LA “COLONIALIDAD DEL SABER"}

Partimos del presupuesto de que históricamente el modelo escolar asimilado por los pueblos indígenas en la América Latina fue el de una escuela homogeneizadora y etnocéntrica pautada por la exclusión de las culturas autóctonas y diferenciadas; pero que en la actualidad se choca con propuestas de una nueva epistemología, la interculturalidad, legitimada por ley.

Así, como veremos en el transcurrir del texto, al conquistar el derecho a una escuela específica y diferenciada, bilingüe, multicultural y comunitaria, los pueblos indígenas abren un campo de estudios en el cual se mueven como protagonistas, en el sentido de pensar el currículo basado en otra lógica: la lógica del diálogo entre sus saberes y los saberes legitimados históricamente por la cultura escolar (AGUILERA URQUIZA, 2010).

Si por un lado, en las aldeas, a través de la resignificación de la escuela, ya constatamos prácticas concretas de una educación intercultural, cuando los indígenas llegan a la enseñanza superior, constatamos por otro lado la existencia de "dos mundos", aquel del conocimiento "científico" y aquel de los saberes 4 indígenas. Anticipamos aquí la propuesta de que ese espacio de la educación superior deje de ser de imposición de una cultura dominantemente eurocéntrica; y

\footnotetext{
${ }^{3}$ Acerca de ese tema, ver: Aguilera Urquiza y Nascimento (2013).

${ }^{4}$ En este texto hacemos referencia al saber y conocimiento como sinónimos, pues entendemos con Mato (2009), que no hay jerarquía entre saber y conocimiento, y si un proceso histórico que descalificó un determinado conocimiento, y que aún se hace presente como una herencia colonial, la colonialidad del saber.
} 
se abra para un espacio intercultural desde el cual emerjan inquietudes y desafíos para todos que lo integre.

A partir del referencial de algunos autores poscoloniales, miramos con extrañeza a la construcción de la diferencia cultural en relación a los occidentales y los pueblos colonizados, a partir de comprensión de la producción de la hegemonía cultural, como consecuencia de las relaciones de poder, ser y saber. Esta perspectiva nos posibilita el entendimiento de que la diferencia es siempre un proceso relacional. Esa diferencia colonial posicionó y legitimó el conocimiento de la Europa, instituyendo en la modernidad la racionalidad científica como el modelo epistemológico global, universal, excluyendo, subalternando o negando la diferencia cultural existente (CALDERONI; AGUILERA URQUIZA, 2018).

Según Lander (2005), el pensamiento científico moderno corroboró para la naturalización de las características de las sociedades occidentales modernas como expresiones del desarrollo histórico de la humanidad y, mismo tras el fin del período colonial, permanecieran favoreciendo aspectos de la cultura europea. Podemos decir que se crio así, la diferencia colonial que marca nuestro pensamiento y nuestras relaciones hasta la actualidad.

Aliados a la modernidad y a la colonialidad los colonizadores se auto representaron; y para allá de la auto representación, construyeron el otro ${ }^{5}$ con bases en las categorías e intereses de su cultura. Esa construcción de la diferencia cultural inventada por los colonizadores sigue fundando las relaciones interculturales en las universidades, con fuerte tradición colonial y eurocéntrica. Las implicaciones, conforme Mato (2009, p. 31), se expresan en las formas con que fueron reproducidas las estrategias de restricción y supresión de "pueblos, comunidades e individuos indígenas y afrodescendientes en los procesos de construcción de los Estados y sociedades nacionales en América Latina [...]".

Para este autor, la estrategia de mayor suceso de los colonizadores fue la invisibilidad de los pueblos colonizados, que es realizada "mediante la omisión de producción de estadísticas tanto acerca de su importancia demográfica en general, como respecto de variables económicas y sociales significativas cruzadas con identificaciones étnicas y/o raciales" (MATO, 2009, p. 31).

\footnotetext{
${ }^{5}$ Los autores del campo conocido como "Modernidad / Colonialidad, recuren a las expresiones como: "pensamiento-otro", conocimiento-otro". Con el "otro", se pretende un cambio de óptica, de lógica, de paradigma (CALDERONI, 2016, p. 24).
} 
Esa invisibilidad es producida intencionalmente, al final, como dicen algunos docentes en clase: "universidad no es espacio de indio. Su lugar es en la aldea". Representaciones como esta demuestran una relación asimétrica, no apenas en relaciones de poder entre personas, pero seguramente entre distintas formas de conocimiento.

Pensamos que esas prácticas eurocéntricas, constatadas en el cotidiano de las universidades, poseen profunda relación con el paradigma de la modernidad. Para comprender esas prácticas eurocéntricas, es necesario buscar sus raíces en la modernidad, la cual presenta, desde sus comienzos, un proyecto emancipador de la razón, de la racionalidad y de la objetividad.

Castro-Gómez (2005, p. 170) afirma que el proyecto de la modernidad pretende poner énfasis en la "formación de los estados nacionales y la consolidación del colonialismo", configurando el conocimiento a partir de varios elementos: el control absoluto del hombre blanco en la conducción del conocimiento con la finalidad de elevar el ser humano al control de todas las cosas; dominio sobre la naturaleza mediante la ciencia; el ser humano bajo el dominio de la razón; la naturaleza como gran adversaria y sumisa al ser humano; el papel de la ciencia someter la naturaleza al control de la razón; la racionalización del occidente como un proceso de desencantamiento del mundo; la ciencia presenta las leyes, el Estado por su vez las utiliza para gobernar; la escrita como dispositivo de poder; la violencia epistémica ocurre en el vínculo entre conocimiento y asignatura (ALVES, 2018, p. 74).

Según Castro-Gómez (2005, p. 170), no se puede comprender el proceso denominado de fin de la modernidad como efecto de la exposición "de los marcos normativos donde el proyecto de la modernidad es jugado 'taxonomicamente', pero si, como una nueva configuración de las relaciones mundiales del poder que ahora son basadas en la representación de las diferencias." A partir de esta perspectiva, podemos decir que la modernidad fue marcada por dos lógicas disciplinares: de un lado la objetividad, y de otro la universalidad, ambas como productoras de un conocimiento correcto y verdadero.

El proyecto moderno se basa en la construcción de un método "universal" para la elaboración del conocimiento. Además de la supuesta racionalidad y objetividad en la construcción de los conocimientos, con pretensión de "verdad" y de "universalidad", podemos destacar, también, otra faz del proyecto de la 
modernidad, la "colonialidad del poder y de saber". Este término utilizado por Quijano (2000), considera la colonialidad uno de los principales elementos constituyentes del modelo mundial del poder capitalista. Para Quijano (2000, p. 359), tal patrón tiene su génesis en el concepto "de una clasificación racional y étnica de la población del mundo como piedra angular de dicho patrón de poder, y opera en cada uno de los planos, ámbitos y dimensiones materiales y subjetivos, de la existencia social cotidiana y la escala societal".

Todos eses elementos de la modernidad expuestos anteriormente se oponen al modo indígena del "buen vivir", sus formas de producción de conocimiento y sus cosmologías indisociables de la naturaleza. Ambivalencias estas que también irán se chocar con la presencia de los pueblos indígenas en los institutos de educación superior, marcados por ese paradigma de la modernidad y de la colonialidad. Reafirmamos, así, que el colonialismo fue más que un sistema de explotación económica y de dominación política, y puede ser entendido como un modo de percepción del mundo y de encuadramiento de la vida social impuesto a los pueblos amerindios, o en las palabras. Este proceso también es denominado por Gruzisnky (2003) como una "colonización del imaginario".

Podemos afirmar, así, en un sentido más amplio, que el colonialismo pasa a ser entendido como un evento que llegó a los dominios más insólitos de las prácticas cotidianas, los más recónditos rincones de la vida social, que pueden ser verificados en las sutilezas del día a día de los sujetos, como el vestuario, los hábitos alimenticios o las formas de entretenimiento. En ese sentido, el colonialismo puede ser entendido como producto de un complejo encuentro que alteró a todos nosotros, como un proceso histórico totalizante, que instituyo una hegemonía del Occidente, orientando incluso nuestra forma de percepción de la realidad.

Pese a esta aparente contraposición entre pueblos indígenas (conocimiento tradicionales) y universidades (conocimientos Ilamados universales), podemos decir que hay una ambivalencia, o sea, en que las partes no son irreductibles, pero posibles de se negociar. Como veremos a continuación, es posible encontrar espacios en la academia para la presencia no solamente física de los pueblos indígenas, pero también, para experiencias y modalidades de colaboración intercultural.

Todavía, el acceso de los indígenas a la enseñanza superior todavía preocupa a las comunidades, sobre todo para las aldeas más lejanas. Sin embargo, actualmente la permanencia se ha vuelto el gran desafío, pues no se trata solo 
de cuestiones financieras, pero principalmente de la necesidad de espacios colaborativos y de diálogo intercultural.

\section{EDUCACIÓN SUPERIOR, PUEBLOS INDÍGENAS Y MODALIDADES DE "COLABORACIÓN INTERCULTURAL"}

Empiezo este apartado, citando la manifestación por escrito de un grupo significativo de estudiantes indígenas reunidos en Campo Grande, en 2011, en el Vo Encuentro de Académicos Indígenas de Mato Grosso do Sul.

O Índio e a Universidade.

Durante muitos anos depois da invasão do Brasil pelos portugueses, muitos dos povos indígenas se limitavam a defender seu povo com arcos, flechas, lanças e outras armas de sua produção. Buscavam fugir do contato com o homem branco nos meios das florestas, escondidos nas matas.

Em certas ocasiões a fuga não representava o sossego de nenhum povo. Então muitos membros de etnias, principalmente as que ficavam na parte central do Brasil, resolveram encarar os problemas de frente e foram morar na cidade.

Em meios aos problemas, da pobreza e da fome, esses indígenas se dedicaram aos livros e bancos escolares. Como

naquele tempo a discriminação, inclusive da educação era grande, onde normalmente as escolas eram apenas para quem tinha dinheiro, as dificuldades enfrentadas por membros de nossos povos eram infinitas.

Muitos comemoraram ao chegar à conclusão do Ensino médio, e retornaram a luta em favor de seu povo.

Outros sonharam mais alto e conquistaram espaços em universidades, se tornaram professores, historiadores, escritores e mestres. Esses engajaram em uma luta

maior, lutar pela liberdade de escolha e não ser tutelado

pela FUNAl, esses foram os primeiros a provar que não precisaríamos de ninguém que falasse por nós, apenas o que precisaríamos era ser ouvidos.

Hoje em dia, a presença indígena nas Universidades é bem maior, por meios de vestibulares regulares ou por meio de ações afirmativas, os índios conquistaram esse espaço e 
fazem dele uma arma de luta no dia a dia. Se antes as armas contra os invasores e destruidores de nossas Terras eram arcos, flechas e lanças, hoje a nossa forma de lutar é no papel e na caneta e como arma maior a justiça. Embora haja dificuldades, muitos Acadêmicos já estão graduados, e já ajudam seus povos. Outros lutam pela permanência e pelo sucesso nos cursos superiores. ${ }^{6}$

En este documento, los estudiantes indígenas expresan las dificultades para cursar la enseñanza superior, reconocen que la presencia indígena está aumentando en la universidad y que esta realidad puede ser comprendida como una nueva herramienta (no más las flechas, pero si el papel y el bolígrafo) en la lucha por autonomía y autodeterminación de eses pueblos.

Sin embargo, sabemos de las dificultades que enfrentan las universidades para incluir eses pueblos indígenas con sus conocimientos propios y flexibilizar los planes de estudio y actividades de cooperación intercultural. Desafortunadamente, todavía prevalece la negación de la alteridad indígena, así como predominan las prácticas de invisibilidad de la presencia indígena, en especial, de negación de sus conocimientos. Entre los derechos rechazados por el sistema educacional occidental se destaca la educación escolar indígena. A pesar de la educación indígena ser asegurada por el Estado brasileño, todavía sigue muy burocratizada (BARROS; ALVARENGA, 2016).

Los autores mencionados aseveran que, "en la práctica, las burocracias y el modus operandi de las universidades brasileñas, con sus vicios positivistas y academicistas, continúan inviabilizando su plena realización." Dicho de otro modo, aunque haya consciencia a respeto de los desaciertos en lo que se refiere a la educación, especialmente bajo la protección de la cultura hegemónica nacional, marcada por su vez por el etnocentrismo y por el eurocentrismo, "escapar de sus dominios cuanto a la educación indígena aun dependerá de mucha lucha y resistencia, visto que el sistema de dominación cultural se encuentra enraizado en la sociedad brasileña, bien como en la academia y en la escuela" (BARROS; ALVARENGA, 2016, p. 311).

Como afirman Brand y Calderoni (2012, p. 181), son pueblos con saberes y procesos sociales e históricos profundamente diferenciados y que traen en común,

\footnotetext{
${ }^{6}$ Actas del Vo Encuentro de Académicos Indígenas de Mato Grosso do Sul. Campo Grande, MS, 2011.
} 
también, una larga trayectoria marcada por la subalternización por otros pueblos, lenguajes y conocimientos. No se trata, por lo tanto, de la inclusión de ese otro, en el caso los académicos indígenas, en el nuestro, en especial, en nuestras lógicas de producción y reproducción de saber, pero de abrir espacios de diálogo para que sus lenguajes y sus procesos de producción de saber puedan ser igualmente legitimados en las instituciones de enseñanza superior. No obstante, para eso, será necesario cuestionar, también, la subalternización históricamente impuesta a eses pueblos.

Insistimos, en este punto, con la propuesta de "modalidades de colaboración intercultural", concepto propuesto por Mato (2017), que va más allá del simples "diálogo de saberes", o sea, son las personas que deben dialogar y cooperar para la superación de los preconceptos. Creemos, así, que incluso en las universidades dichas tradicionales, es posible encontrar brechas y mecanismos para impulsar prácticas de interculturalidad.

Según Fleury (2003, p. 17), el concepto de interculturalidad viene siendo utilizado para ajustar realidades y perspectivas que divergen entre sí. La interculturalidad se refiere, por lo tanto, a la posibilidad de promoción de la coexistencia entre distintas culturas, evitando y apaciguando conflictos. Busca relaciones simétricas entre las culturas y no la superioridad, incentiva relaciones cooperativas y respeta las identidades culturales de cada una.

Vieira (2001, p. 118), complementa esta definición afirmando: "la interculturalidad no busca la hegemonía, más el reconocimiento de la diversidad" y la negociación de las diferencias, sin relaciones jerárquicas de poder. Considerando que la universidad pública es para todos/as, se supone que ella sea ofrecida indistintamente, y no como venía ocurriendo, en el caso brasileño, antes de 2012, cuando fue sancionada la Ley Federal n. 12.711.

El año de 2012, según Bergamaschi, Doebber y Brito (2018), se convirtió en un marco de una nueva relación del Estado brasileño con la política de acciones afirmativas generales, incluso para los pueblos indígenas, en universidades federales e instituciones federales de enseñanza técnico de nivel medio. La promulgación de la Ley n. 12.711, de 29 de agosto de 2012, intitulada Ley de Cupos, encierra la discusión acerca del mérito de la implantación de mecanismos de acceso y permanencia de alumnos autodenominados negros e indios en las universidades federales brasileñas, instituyendo el carácter obligatorio de la adopción de 
tales políticas, que, anteriormente, dependían de la iniciativa de cada institución (BERGAMASCHI; DOEBBER; BRITO, 2018, p. 40).

Esa universalización aunque parezca positiva, no asegura la discusión sobre la permanencia de los indígenas en la educación superior, la cual pasa en grande medida, por el reconocimiento de sus conocimientos y de las prácticas efectivas de colaboración intercultural. En este sentido, afirma Candau (2008a, p. 50),

En el caso de la educación, se promueve una política de universalización de la escolarización, todos son llamados a participar del sistema escolar, pero no se pone en discusión el carácter monocultural presente en su dinámica, tanto en lo que se refiere a los contenidos del currículo cuanto a las relaciones entre los distintos actores, a las estrategias utilizadas en las clases, a los valores privilegiados etc. Únicamente, los que no tenían acceso a eses bienes y a esas instituciones son incluidos en ellas tal como son. Esa posición defiende el proyecto de construir una cultura común y, en nombre de ella, deslegitima dialectos, saberes, lenguas, creencias, valores "distintos", pertenecientes a los grupos subordinados, considerados inferiores explícita o implícitamente.

Ante esa constatación, apostamos en la construcción de un modelo intercultural de educación, enfocado en la participación de las comunidades indígenas, en las particularidades y especificidades de las diferentes culturas. Cabe resaltar que la lucha por la política de acciones afirmativas tuvo la presencia actuante del movimiento indígena. No obstante, la implementación de estas iniciativas en las universidades, viene ocurriendo dentro de la comunidad académicos, muchas veces con agremiaciones de profesores sin ninguna experiencia con pueblos indígenas.

Por otro lado, es necesario que estas políticas aparezcan en los planes de estudios y que los cursos de grado y posgrado se abran para el intercambio de experiencias, para acoger ese "otro", radicalmente diferente de nuestra cultura moderna y eurocéntrica. Todavía respecto a esa propuesta, Candau (2008a) elabora algunas consideraciones acerca de la perspectiva intercultural, en las cuales enfatiza la necesidad urgente de promoción de una educación direccionada para el reconocimiento de la alteridad que caracteriza el "otro" para que se pueda fomentar el diálogo entre agrupamientos socioculturales distintos. Para Candau, la constitución de un proyecto colectivo, en que las divergencias puedan ser integradas de forma dialéctica, exige "Una educación para la negociación cultural, 
que afronte los conflictos provocados pela asimetría de poder entre los diferentes grupos socioculturales" (CANDAU, 2008a, p. 52).

La Constitución Federal brasileña, de 1988, y el ordenamiento jurídico infra constitucional, garantizan a los pueblos indígenas el acceso a una educación específica, diferenciada, comunitaria, bilingüe e intercultural, la cual debe valorizar sus lenguas, culturas y tradiciones. En ese sentido, afirmamos que ese marco legislativo planteó el comienzo de una educación intercultural con la intención de reducir el desfasaje escolar de las comunidades indígenas para, a partir de esta situación de transición, poder contribuir para cambiar la mentalidad dominante (tradicional) y ofrecer una respuesta a la realidad multicultural del país.

Llegamos así, una vez más, al centro del tema abordado en el presente texto: las aldeas, de manera general, consiguen desarrollar prácticas de educación intercultural, incluso porque la gran parte de los profesores es indígena. Esa realidad cambia radicalmente cuando los/as jóvenes indígenas llegan a la educación superior: espacio de un pensamiento único, eurocéntrico, enyesado en "planes de estudios", y sin espacios para diálogos y reconocimiento de las culturas autóctonas.

Esa realidad, conforme Brand y Calderoni (2012), demanda una reflexión pormenorizada de las formas de emergencia, en la América, de las estructuras formativas que caracterizaran los procesos de colonización; que descaracterizaron, subvirtieran e interiorizaran sociedades amerindias y sus conocimientos tradicionales. Para eso, la estrategia utilizada fue imponer a eses pueblos la forma de saber europea, representada de forma eurocéntrica como universal. "No se trata", concluyen los autores, "de una cuestión cronológica, pues, ciertamente, la América hoy no es más colonia de Europa, pero si de una revisión epistémica, que pasa por el cuestionamiento de las relaciones de poder, que siguen caracterizadamente coloniales" (BRAND; CALDERONI, 2012, p. 182).

Postulamos, así, esta revisión epistémica de las prácticas coloniales aún presentes en la educación superior en Latino América, especialmente en Brasil, alejando los conocimientos de jóvenes indígenas, afrodescendientes y de otras minorías. Solamente una universidad abierta para acoger el "otro", implementar propuestas y modalidades de "colaboración intercultural", podrá dar respuestas convincentes a las demandas actuales de los pueblos indígenas y su derecho a una educación pública y de cualidad y que respete sus conocimientos. 


\section{EDUCACIÓN SUPERIOR, PUEBLOS INDÍGENAS Y LA PRODUCCIÓN DE CONOCIMIENTOS}

Cuando los indígenas llegan a las universidades, muchas veces traen consigo algunos déficits de aprendizaje, o de contenidos no estudiados, o incluso de prácticas metodológicas exigidas en la educación superior y de difícil acceso, para quien viene de una tradición de oralidad. Sin embargo, todas esas dificultades iniciales, suelen ser superadas, cuando es ofrecido a los académicos autóctonos un acompañamiento pedagógico basado en el respeto a sus historias y sus realidades culturales y surgen oportunidades para procesos colaborativos entre sociedad indígena y no indígena.

Respecto a los posibles déficits que algunos individuos indígenas puedan presentar acerca de la aprehensión de contenidos valorizados por la educación impuesta por la sociedad nacional. Creemos que la solución se encuentra en la educación básica, ya que es esta la responsable por la formación de jóvenes indígenas en las cuestiones relacionadas al desarrollo de competencias y habilidades consideradas relevantes a la sociedad nacional.

Tras más de diez años de experiencia con el Programa Rede de Saberes (AGUILERA URQUIZA; NASCIMENTO, 2013), es posible apostar en la posibilidad de empoderar a los estudiantes indígenas dentro de las universidades. Para eso, es importante la preparación de los profesores, los técnicos y la propia universidad para recibir estos pueblos tradicionales y para el intercambio intercultural.

Las universidades que participan de las acciones afirmativas, acogiendo indígenas y afrodescendientes en Brasil, también se transforman, ya que tienen que abrirse a las nuevas epistemologías, ontologías y sujetos. De este modo, establecen espacios de nuevas sociabilidades, especialmente, entre indígenas y no indígenas. En consecuencia, pasan por transformaciones incluso en sus espacios más convencionales, a partir de la relación establecida con los diversos departamentos y programas de pos grado. Todo eso promueve nuevos conocimientos contemplados en disertaciones y tesis enfocados en el tema como objeto de estudio y favorecen la creación de nuevas políticas, como por ejemplo las relacionadas a acciones afirmativas en el posgrado de algunas universidades brasileñas.

Sin embargo, es necesario avanzar aún más y abandonar en el pasado las prácticas de una educación superior direccionada para la formación de la elite 
blanca de nuestros países. Permanece el desafío de que no favorecer el acceso a la universidad por las sociedades indígenas es primordial pensar en políticas de permanencia y construir prácticas interculturales de producción de conocimientos.

A partir de esta perspectiva, Brand y Calderoni (2012, p. 182) afirman que es importante reflexionar sobre como eliminar la jerarquización, marginalización y subalternización que caracteriza la influencia y la relación de la ciencia oficial con las diversas otras formas de conocimiento; o dicho de otra manera: dar voz e vez a concepciones socioculturales de mundo estratégicamente alejadas y reprimidas por el proyecto colonial.

Como ya es sabido, promover el acceso de indígenas a la enseñanza superior no es suficiente para eliminar la subalternidad, considerando que la mayoría de los programas elaborados para facilitar el acceso al conocimiento moderno, tiene por objetivo la manutención de la inferiorización de toda y cualquier otra forma de conocimiento.

Como el proceso de democratizar la educación superior está en marcha en toda Latino América, el desafío mayor en la actualidad es "interculturalizar y descolonizar la educación superior" (MATO, 2016, p. 41), a través de mecanismos que devuelvan la voz a los pueblos indígenas dentro de las universidades, que sus conocimientos tengan relevancia y que puedan demonstrar su capacidad en la construcción de nuevos conocimientos.

Para Freire (2005), la palabra tiene el papel de pronunciar el mundo, de problematizar, y de modificar ese mundo. El papel de la palabra como acción, se acerca del argumento de la perspectiva poscolonial - en la cual el lenguaje y el discurso tienen lugar central (PENNA, 2014, p. 193). El lenguaje, de esa forma, es una práctica de colectividad, nadie podrá decir solo, o según Freire (2005, p. 91) "nadie puede decir la palabra verdadera solo, o decir para los otros, en un acto de prescripción, con el cual roba la palabra de los demás".

A partir de este punto de vista, según Penna (2014, p. 193) podemos percibir cierta relación entre Paulo Freire y los autores poscoloniales en lo que se refiere a la superación de la dominación/eurocentrismo:

La "Pedagogía del Oprimido" propone la objetivación y la desconstrucción del mito de la estructura opresora como estrategia para que el educando pueda cuestionar temas y aspectos de la realidad antes considerados como dados, superando así su visión fatalista del mundo. De manera semejante la 
literatura poscolonial aboga por la objetivación y desconstrucción del mito del eurocentrismo, a través del paradigma de la colonialidad/modernidad, como forma de producir un conocimiento menos colonizado y excluyente; con carácter pedagógico proveniente del campo de las ciencias sociales.

Así, una de las estrategias de construcción de la autodeterminación de los pueblos indígenas ocurre a través de prácticas de interculturalidad que prevé relaciones simétricas y de respecto con las diferencias. Según Penna (2014, p. 1934), aunque exista divergencias entre los pensadores poscoloniales (decoloniales) acerca del cual debe ser la salida para el problema de la "colonización del ser", se puede decir que todos convergen cuanto al argumento de que el poscolonialismo representa la inserción de un nuevo lugar de habla, de un nuevo lugar de enunciación del punto de vista de la geopolítica del conocimiento.

\section{CONSIDERACIONES FINALES}

Cuando tratamos del eurocentrismo en la educación superior y exaltamos la necesidad de su abertura para las minorías, nos deparamos con la realidad de algunas políticas públicas que "pocas veces incorporan las demandas y propuestas formuladas por las organizaciones indígenas y afrodescendientes" (MATO, 2016, p. 42).

En el caso de las universidades, es insuficiente las acciones en favor de la inclusión de indígenas y negros en la educación superior, si no viene acompañada de políticas de permanencia que va más allá de la actual "beca permanencia", en el caso brasileño.

Según los/as propios/as estudiantes indígenas, son necesarias otras acciones, como por ejemplo: espacio de acogida y convivencia de estos grupos, espacios de informática, momentos de manifestaciones culturales y autoafirmación cultural, momentos de monitoria y acompañamiento del aprendizaje académica, espacio/estímulo para iniciación científica, apoyo psicológico, además de infraestructura (habitación, alimentación, transporte), etc. Son demandas presentadas por aquellos/as que en la actualidad están estudiando la enseñanza superior en la Universidad Federal de Mato Grosso do Sul (UFMS), Brasil, que se asemejan a otros pedidos realizados por estudiantes indígenas de otros países latinoamericanos.

A pesar de todas esas limitaciones, concluimos que la educación superior puede ser y está se transformando en un espacio de construcción de autonomía 
de eses pueblos y de valorización de sus conocimientos tradicionales. Para las comunidades indígenas, las universidades que buscan "acoger" la diferencia, están se constituyendo como un "tercero espacio", o sea, tienen demandado esfuerzos epistemológicos y metodológicos para evitar la política de la polaridad (cultura alta $X$ cultura baja) y están permitiendo el surgimiento en cuanto "otros de ellos mismos" (BHABHA, 1998).

Para Lopes (2005, p. 11), "se les está permitiendo tomar conciencia de la posibilidad de considerar formas de reencontrarse consigo mismos y de imaginar alternativas educativas que den cuenta de su condición diferente y diferenciada dentro del contexto". En otras palabras, el acceso y la permanencia de indígenas en la educación superior dependerán principalmente de la capacidad institucional de proponer modalidades de colaboración intercultural, para que eses espacios se tornen estratégicos para la construcción de la autonomía de estos pueblos.

Entendemos, así, que a pesar de la tendencia al etnocentrismo eurocéntrico, las universidades no solamente pueden dejar de reproducir las prácticas de exclusión y de preconcepto contra los pueblos indígenas, pero también pueden se transformar en espacios privilegiados para el diálogo, el encuentro de culturas y la colaboración intercultural. A partir de una perspectiva epistemológica y política seria asumir "las diferencias como constitutivas de la democracia y [se tornaren] capaces de construir nuevas relaciones (CANDAU, 2009, p. 9).

Esas nuevas relaciones no son propuestas dirigidas exclusivamente para los pueblos indígenas, y afrodescendientes, sino corresponde una alternativa intercultural para todas las culturas de forma general. En ese sentido, el acceso y la permanencia de indígenas en la enseñanza superior podrá servir como herramienta para abandonar las tendencias monoculturales de las universidades y del propio Estado brasileño en sus intentos de homogeneización, promoviendo de este modo la autonomía regional y las políticas de sustentabilidad.

\footnotetext{
7 Director del PROEIB/ANDES- Programa de formación en Educación Intercultural Bilingüe para los Países Andinos. Cochabamba/Bolivia.
} 
Educación superior en Brasil y modalidades de colaboración intercultural - espacio estratégico de construcción de la autodeterminación de los pueblos indígenas

\section{REFERENCIAS}

AGUILERA URQUIZA, Antonio Hilário. Educação escolar indígena no Brasil: os caminhos de uma guinada política e epistemológica. In: NASCIMENTO, Claudemiro Godoy do (Org.). $O$ verso e o reverso da educação - das políticas às pedagogias alternativas. 1. ed. Goiânia, GO: Editora PUC-GO, 2010. v. 01, p. 137-66.

AGUILERA URQUIZA, Antonio Hilário; NASCIMENTO, Adir C. Rede de saberes - políticas de ação afirmativa no Ensino Superior para indígenas no Mato Grosso do Sul. 1. ed. Rio de Janeiro: FLACSO, GEA; UERJ, LPP, 2013. v. 01, 86p.

ALVES, Maria Isabel Alonso. Narrativas de professoras indígenas Arara (Karo Rap) de Rondônia: identidades entre experiências formativas escolares e não escolares. 2018. Tesis (Doctorado en Educación) - Universidade Católica Dom Bosco (UCDB), Campo Grande, MS, 2018.

BARROS, Ana Maria; ALVARENGA, Rodrigo. Os povos indígenas brasileiros e sua luta por uma educação específica, diferenciada e intercultural. In: LIMA, Cezar Bueno; GUEBERT, Mirian Célia Castellain (Org.). Teorias dos direitos humanos em perspectiva interdisciplinar. Curitiba, PR: PUCPRess, 2016.

BERGAMASCHI, Maria Aparecida; DOEBBER, Michele Barcelos; BRITO, Patrícia Oliveira. Estudantes indígenas em universidades brasileiras: um estudo das políticas de acesso e permanência. Revista Brasileira de Estudos Pedagógicos, Brasília, v. 99, n. 251, p. 37-53, jan./abr. 2018.

BHABHA, Homi. O local da cultura. Belo Horizonte, MG: UFMG, 1998.

BRAND, Antonio J; CALDERONI, Valéria A. M. de Oliveira. Povos indígenas, conhecimentos tradicionais e diálogo de saberes nas instituições de educação superior: desafios para uns e outros. Série-Estudos, Campo Grande, MS, n. 34, p. 179-88, jul./dez. 2012.

CALDERONI, Valéria Aparecida Mendonça de Oliveira. Professores indígenas e educação superior: traduções e negociações na Escola Indígena Ñandejara da aldeia Te'ýikue, Caarapó/MS. 2016. 305f. Tesis (Doctorado en Educación) - Universidade Católica Dom Bosco (UCDB), Campo Grande, MS, 2016.

CALDERONI, Valéria Aparecida Mendonça de Oliveira; AGUILERA URQUIZA, Antonio Hilário. Dialogando sobre interculturalidade e educação, abordagens interculturais indígenas. Texto presentado-publicado em el ICA (International Congresso of Americanists), Salamanca, España, 2018. 
CANDAU, Vera Maria (Org.). Educação intercultural na América Latina: entre concepções, tensões e propostas. Rio de Janeiro: 7 Letras, 2009.

CANDAU, Vera Maria. Direitos humanos, educação e interculturalidade: as tensões entre igualdade e diferença. Revista Brasileira de Educação, Rio de Janeiro, v. 13, n. 37, p. 4556, jan./abr. 2008a.

CASTRO-GÓMEZ, Santiago. Ciencias sociales, violencia epistémica y el problema de la "invención del otro". In: LANDER, Edgardo (Org.). A colonialidade do saber: eurocentrismo e Ciências Sociais. 1. ed. Buenos Aires: CLACSO, 2005.

FREIRE, Paulo. Pedagogia do oprimido. 47. ed. Petrópolis, RJ: Vozes, 2005.

FLEURY, Reinaldo Matias. Educação e intercultura. Revista Brasileira de Educação, Rio de Janeiro, n. 23, p. 16-35, maio/ago. 2003. Disponível em: http://www.scielo.br/pdf/rbedu/ n23/n23a02.pdf. Acesso em: 15 jul. 2017.

GRUZINSKI, Serge. Colonização do imaginário. Tradução Beatriz Perrone-Moisés. São Paulo: Companhia das Letras, 2003.

LANDER, Edgardo (Org.). A colonialidade do saber: eurocentrismo e Ciências Sociais. 1. ed. Buenos Aires: CLACSO, 2005.

LÓPEZ, Luis Enrique. Prefácio. In: CARIMAN, Geraldine Abarca. Rupturas y continuidades en la recreación de la cultura mapuche en Santiago de Chile. La Paz, Bolívia: Plural Editores, 2005. p. 11-4.

MATO, Daniel. Del "diálogo de saberes" a la construcción de modalidades de "colaboración intercultural": aprendizajes y articulaciones más allá de la Academia. Revista Lasa Forum, v. XLVIII, n. 3, p. 8-17, summer 2017.

MATO, Daniel. Universidades e diversidade cultural e epistêmica na América Latina: experiências, conflitos e desafios. In: CANDAU, V. M. (Org.). Interculturalizar, descolonizar, democratizar: uma educação "outra"? Rio de Janeiro: 7 Letras, 2016. p. 38-63.

MATO, Daniel. Diversidad cultural e interculturalidad en educación superior. Problemas, retos, oportunidades y experiencias en América Latina. In: MATO, Daniel (Coord.). Diversidad cultural e interculturalidad en educación superior: experiencias en América Latina. México: Colección Cuadernos Interculturales/Calle, 2009.

MELIÁ, Bartomeu. Educação indígena e alfabetização. São Paulo: Loyola, 1979. 
PENNA, Camila. Paulo Freire no pensamento decolonial: um olhar pedagógico sobre a teoria pós-colonial latino-americana. Revista de Estudos \& Pesquisas sobre as Américas, Brasília, v. 8, n. 2, p. 181-99, 2014.

QUIJANO, Aníbal. Colonialidad del poder, clasificación social. Journal of World - Systems Reserche, v. XI, n. 2, p. 342-86, 2000.

VIEIRA, Rosangela Steffen. Educação intercultural: uma proposta de ação no mundo multicultural. In: FLEURI, R. M. (Org.). Intercultura: estudos emergentes. Florianópolis, SC: Mover; Ijuí, RS: Editora Unijuí, 2001. p. 117-27.

\section{Sobre el autor:}

Antonio Hilário Aguilera Urquiza: Doctorado en Antropología. Profesor de los cursos de Postgrado en Antropología social y en Derecho, de la Universidade Federal de Mato Grosso do Sul (UFMS) y profesor colaborador del curso de Postgrado en Educación de la Universidade Católica Dom Bosco (UCDB). E-mail: hilarioaguilera@gmail.com

\section{Recebido em maio de 2018 Aceito em julho de 2018}


